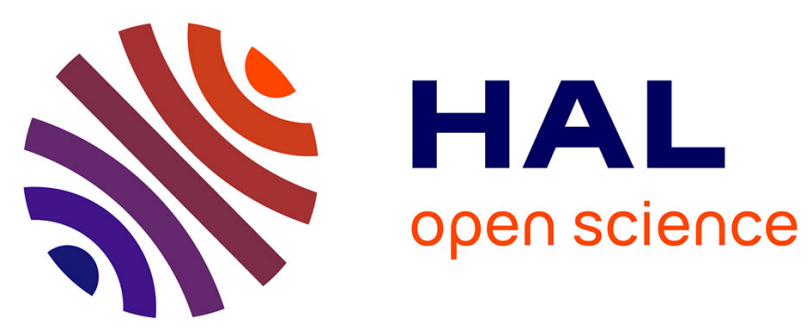

\title{
Application of Blind Source Separation Methods to Ion-Selective Electrode Arrays in Flow-Injection Analysis
}

Leonardo Tomazeli Duarte, João Marcos Romano, Christian Jutten, Karin Chumbimuni-Torres, Lauro Kubota

\section{To cite this version:}

Leonardo Tomazeli Duarte, João Marcos Romano, Christian Jutten, Karin Chumbimuni-Torres, Lauro Kubota. Application of Blind Source Separation Methods to Ion-Selective Electrode Arrays in FlowInjection Analysis. IEEE Sensors Journal, 2014, 14 (7), pp.2228-2229. 10.1109/JSEN.2014.2318174 . hal-01009910

\section{HAL Id: hal-01009910 https://hal.science/hal-01009910}

Submitted on 7 Jul 2014

HAL is a multi-disciplinary open access archive for the deposit and dissemination of scientific research documents, whether they are published or not. The documents may come from teaching and research institutions in France or abroad, or from public or private research centers.
L'archive ouverte pluridisciplinaire HAL, est destinée au dépôt et à la diffusion de documents scientifiques de niveau recherche, publiés ou non, émanant des établissements d'enseignement et de recherche français ou étrangers, des laboratoires publics ou privés. 


\title{
Application of Blind Source Separation Methods to Ion-selective Electrode Arrays in Flow-injection Analysis
}

\author{
Leonardo T. Duarte, Member, IEEE, João M. T. Romano, Senior Member, IEEE, Christian Jutten, Fellow, IEEE, \\ Karin Y. Chumbimuni-Torres, and Lauro T. Kubota
}

\begin{abstract}
As shown recently, the interference problem typical of ion-selective electrodes can be dealt with via smart arrays adjusted by blind source separation (BSS) methods. In this letter, we resume this study and show that such an approach can be applied even when faced with a limited number of samples acquired through flow-injection analysis.
\end{abstract}

Index Terms-Chemical sensor arrays, blind source separation, ion-selective electrodes, signal processing.

\section{INTRODUCTION}

Although providing a simple solution for measuring ionic activity, an ion-selective electrode (ISE) often lacks selectivity, i.e., its response may be influenced by ions other than the target one. A possible solution to this problem is to set up an array composed of different ISEs. This allows the desired information to be recovered by signal processing methods that exploit the diversity provided by the array (diversity here means that each sensor responds differently). In this context, unsupervised signal processing methods can be particularly useful, since it works without calibration, or, at least, with a limited number of training samples. Such a feature can be interesting in ionic analysis since it avoids the need for performing calibration from time to time.

In the context of ISE arrays, an unsupervised approach for quantitative analysis leads to a blind source separation (BSS) problem [1], whose goal is to estimate a set of source signals by only considering mixtures of these sources. In ISE arrays, the sources and the mixtures are the activities of each ion within the solution and the responses provided by the ISE array, respectively. Among the difficulties in the application of BSS methods to ISE arrays is the fact that the mixing process is nonlinear. Indeed, ISEs are often modeled by the NicolskyEisenman (NE) equation, in which the response of the $i$-th sensor within the array is given by:

$$
x_{i}(t)=e_{i}+d_{i} \log _{10}\left(s_{i}(t)+\sum_{j=1, j \neq i}^{N_{s}} a_{i j} s_{j}(t)\right),
$$

L.T. Duarte is with the School of Applied Sciences (FCA), University of Campinas (UNICAMP), Limeira, Brazil (leonardo.duarte@fca.unicamp.br).L. T. Duarte thanks the Fapesp for funding his research. This work has been partly supported by the European project ERC-2012-AdG-320684-CHESS.J. M. T. Romano is with the School of Electrical and Computer Engineering (FEEC), University of Campinas (UNICAMP), Campinas, Brazil (email: romano@dmo.fee.unicamp.br). C. Jutten is with the GIPSA-lab (UMR CNRS 5216), Institut Polytechnique de Grenoble, Grenoble, France (e-mail: christian.jutten@gipsa-lab.grenoble-inp.fr), and also with the Institut Universitaire de France. K. Y. Chumbimuni-Torres is with the University of Central Florida (UCF), Orlando, FL (e-mail: karin.chumbimunitorres@ucf.edu). L. T. Kubota is with the Institute of Chemistry (IQ), University of Campinas (UNICAMP), Campinas, Brazil (e-mail: kubota@iqm.unicamp.br). where $s_{i}(t)$ and $s_{j}(t)$ are the activity of the target and interfering ions, respectively; $e_{i}$ and $d_{i}$ are constants and $a_{i j}$ are the selectivity coefficients. $N_{s}$ is the number of sources and can be estimated by model order selection methods.

In a recent work [2], a nonlinear BSS method based on a Bayesian approach was applied to ISE arrays. The results were encouraging and showed that BSS methods indeed provided good results for actual data. Herein, we extend this study by considering a more realistic scenario in which the data was acquired via a flow-injection analysis yielding a limited number of samples. Concerning the BSS method, we apply the Bayesian solution of [2], but also a BSS method based on independent component analysis (ICA) [3].

\section{Methodology}

\section{A. Data acquisition}

The data was acquired by an ISE array composed of two electrodes tailored to sodium $\left(\mathrm{Na}^{+}\right)$and potassium $\left(\mathrm{K}^{+}\right)$ions, respectively. Moreover, a flow injection analysis system (FIAs) was considered. In a FIAs, the solution under analysis is pumped through a tube system allowing them to be exposed to the sensors in a continuous sampling. There may be several successive injections of the same solution. There are several advantages in considering a FIAs. It presents a low-cost procedure in which a small quantity of reagent is necessary, and offers a more reproducible and rapid analysis compared to traditional beaker analysis.

In the performed experiments, 41 standard solutions were analyzed. The activities of $\mathrm{Na}^{+}$and $\mathrm{K}^{+}$for each of these solutions are shown in Figure 1 (these 41 samples can be represented as two signals which correspond to the desired sources). Concerning the FIAs, three successive injections for each standard solution were performed. There are thus three peaks associated with a given standard solution. The ISE array response for a given standard solution were obtained by averaging the three peaks associated with such solution. The obtained signals, which correspond to the mixtures in our problem, are shown in Figure 2.

\section{B. BSS methods}

Two BSS methods were applied to estimate the ionic activities depicted in Figure 1 from the mixtures of Figure 2: an ICA-based algorithm and a Bayesian algorithm. In ICA, the central assumption is that the sources can be modeled as statistically independent random variables [1]. Since the mixing process renders the mixtures dependent, ICA estimates the sources by adjusting a separating system whose inputs are 

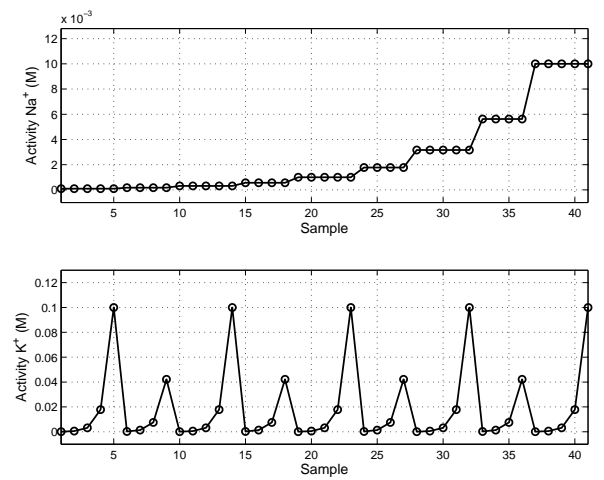

Fig. 1. Sources: activies of $\mathrm{Na}^{+}$and $\mathrm{K}^{+}$.
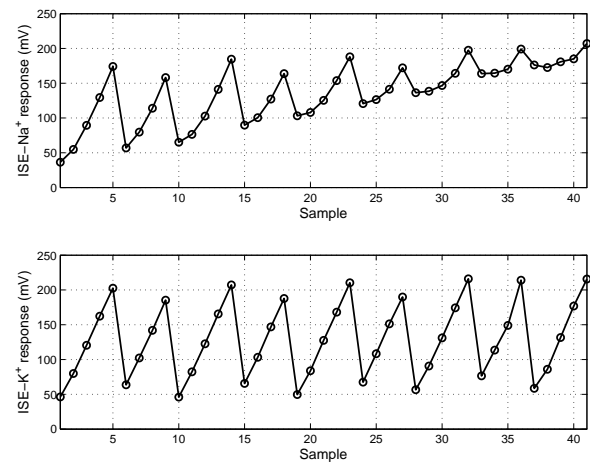

Fig. 2. Mixtures: responses of the $\mathrm{Na}^{+}$and $\mathrm{K}^{+}$ISEs.

the mixtures and the outputs are as independent as possible signals. We considered the ICA algorithm proposed in [3], which provides an efficient solution for the class of mixing systems known as Post-Nonlinear (PNL) models - when the valences of the ions under analysis are equal, the NE equation becomes a particular case of the PNL model [2]. The details of the ICA method adopted here can be found in [3].

In the Bayesian approach, BSS is rather seen as an inverse problem for which a good representation of the data is searched according to the Bayes' rule. In this letter, we adopted the Bayesian BSS algorithm proposed in [2], which is based on a Markov Chain Monte Carlo (MCMC) simulation and is also tailored to PNL models. A Bayesian method can be particularly useful when only a small number of samples is available and when the sources are correlated (see [2] for more details and for an example of correlated sources).

\section{RESULTS}

To compare the BSS methods, the signal-to-distortion (SDR) was considered [4]:

$$
\mathrm{SDR}_{i}=10 \log \left(E\left\{s_{i}^{2}\right\}\left(E\left\{\left(s_{i}-\hat{s_{i}}\right)^{2}\right\}\right)^{-1}\right)
$$

where $s_{i}$ and $\hat{s}_{i}$ denote the source $i$ and its respective estimation after correct scaling (the scale ambiguity, typical of BSS methods, was corrected by using 5 calibration points).

The sources retrieved by the ICA and Bayesian methods are shown in Figures 3 and 4, respectively (the actual sources are plotted in gray). The mixture SDRs, i.e. before any processing, were $\mathrm{SDR}_{1}=1.2 \mathrm{~dB}$ and $\mathrm{SDR}_{2}=4.7$. The obtained SDRs
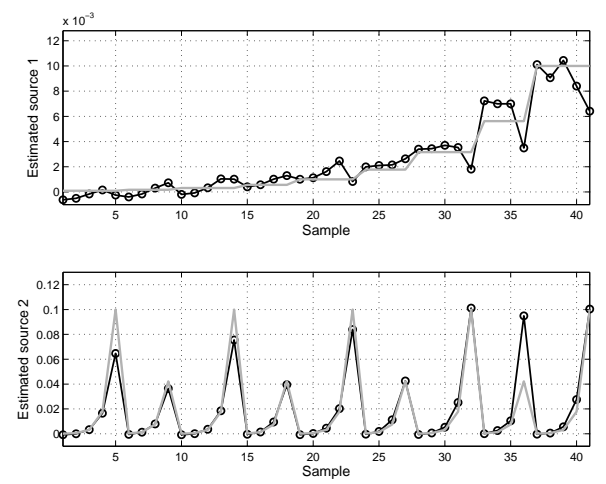

Fig. 3. Sources estimated by the ICA method.
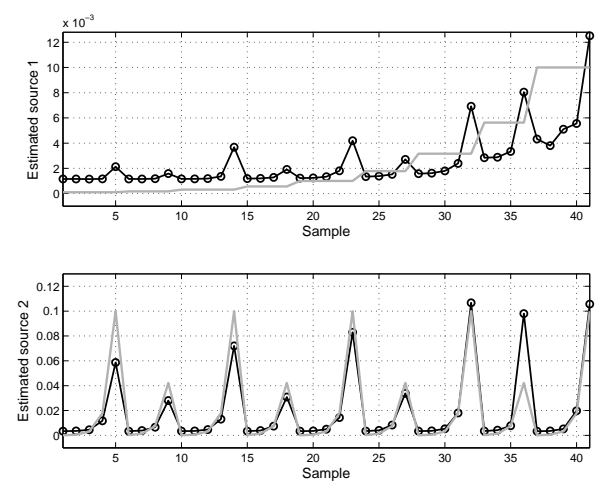

Fig. 4. Sources estimated by the Bayesian method.

for the two sources were $\mathrm{SDR}_{1}=11.0 \mathrm{~dB}$ and $\mathrm{SDR}_{2}=10.6$ $\mathrm{dB}(\mathrm{ICA})$, and $\mathrm{SDR}_{1}=4.4 \mathrm{~dB}$ and $\mathrm{SDR}_{2}=9.3 \mathrm{~dB}$ (Bayesian method). The improvement was between 5 to $10 \mathrm{~dB}$ with ICA while only between 3 to $4.5 \mathrm{~dB}$ with the Bayesian approach.

\section{CONCLUSIONS}

In this work, we applied BSS algorithms in ISE arrays operating in FIA. Despite the limited number of samples, a good estimation of the ionic activities was provided by BSS. However, in contrast to previous work [2], the ICA-based solution provided a better response in comparison to the Bayesian method. Indeed, differently from the data considered in [2], the sources in the present work were highly uncorrelated, which is in accordance with the main assumption of ICA methods. As a perspective for this work, we are currently studying its extension to scenarios in which the number of sources and sensors is greater than two.

\section{REFERENCES}

[1] P. Comon and C. Jutten, Eds., Handbook of blind source separation: independent component analysis and applications. Academic Press, 2010.

[2] L. T. Duarte, C. Jutten, and S. Moussaoui, "A Bayesian nonlinear source separation method for smart ion-selective electrode arrays," IEEE Sensors Journal, vol. 9, no. 12, pp. 1763-1771, 2009.

[3] L. T. Duarte, R. Suyama, R. R. de Faissol Attux, F. J. Von Zuben, and J. M. T. Romano, "Blind source separation of post-nonlinear mixtures using evolutionary computation and order statistics," in ICA and Blind Signal Separation. Springer, 2006, pp. 66-73.

[4] E. Vincent, R. Gribonval, and C. Févotte, "Performance measurement in blind audio source separation," IEEE Transactions on Audio, Speech, and Language Processing, vol. 14, no. 4, pp. 1462-1469, 2006. 